\title{
Relación Entre Clima Organizacional y Engagement, en Dos Fundaciones Sociales, Sin Fines de Lucro, de la Región del Bio Bio
}

\section{RELATIONSHIP BETWEEN ORGANIZATIONAL CLIMATE AND ENGAGEMENT, IN TWO SOCIAL FOUNDATIONS, WITHOUT PROFIT, FROM THE BIO BIO REGION}

\author{
María Margarita Chiang Vega', Ivonne Ingrid Fuentealba Elgueta ${ }^{2}$, Rossana Alejandra Nova Cabrera ${ }^{2}$ \\ 1. Docente Facultad de Ciencias Empresariales, Universidad del Bio Bio, Concepción, Chile. \\ 2. Facultad de Ciencias Empresariales, Universidad del Bio Bio, Concepción, Chile.
}

\begin{abstract}
RESUMEN
El clima organizacional y la presencia de engagement en los trabajadores se constituyen en factores determinantes para la salud laboral de la organización, razón por la cual se presenta un estudio descriptivocorrelacional, que busca asociación entre las variables del clima organizacional y el engagement. La investigación tuvo por objetivo el realizar un análisis de esta relación en dos fundaciones sociales, sin fines de lucro, de la Región del Bio Bio. El Universo de estudio corresponde a 322 trabajadores. El diseño de la muestra está compuesto por 237 trabajadores, lo que equivale al 73,6\% del total del universo de estudio, de los cuales 83 pertenecen a la Fundación 1 y 154 a la Fundación 2. El muestreo fue no probabilístico por conveniencia, pues incluyó la población total de ambas fundaciones. Para efectos de la recolección de la información se utilizó la escala de medición para clima organizacional de Koys \& Decottis ${ }^{1}$ adaptada por Chiang et $\mathrm{al}^{2,3}$, y para medir engagement se aplicó un instrumento de Bienestar y Trabajo (UWES), traducción del Instrumento de Schaufeli y Bakker ${ }^{4}$, Benevides-Pereira, A., Fraiz, D., Porto-Martins, P., versión en español. Para el análisis de la fiabilidad de ambos cuestionarios, se utilizó el alfa de Cronbach.

Los resultados, obtenidos del análisis de datos mediante el coeficiente de Spearman, indicaron que existe asociación estadística significativa entre algunas dimensiones del clima organizacional con algunas dimensiones de engagement de los trabajadores de ambas fundaciones, comprobando así la hipótesis planteada de investigación: "A mayor engagement entre los trabajadores de las fundaciones investigadas se presenta un clima organizacional más adecuado para el logro de los objetivos de cada institución". No obstante, comparando las correlaciones de ambas muestras, se observa que las correlaciones que se dan entre una y otra institución no son las mismas, por lo que no se pueden realizar conclusiones generales.
\end{abstract}

(Chiang M, Fuentealba I, Nova R, 2017. Relación Entre Clima Organizacional y Engagement, en Dos Fundaciones Sociales, Sin Fines de Lucro, de la Región del Bio Bio. Cienc Trab. May-Ago; 19 [59]: 105-112).

Palabras claves: CLIMA ORGANIZACIONAL Y ENGAGEMENT.

\section{ABSTRACT}

The organizational climate and the presence of worker engagement constitute determinants for the organization's occupational health, which is why a descriptive-correlational study is presented, looking for an association between the variables of organizational climate and engagement. The objective of the research was to conduct an analysis of this relationship in two non-profit social foundations of the Bio Bio Region. The study universe corresponds to 322 workers. The design of the sample is made up of 237 workers, equivalent to $73.6 \%$ of the total study universe, of which 83 belong to Foundation 1 and 154 to Foundation 2. Sampling was non-probabilistic for convenience, as it included The total population of both foundations. For the purposes of information collection, the Koys \& Decottis ${ }^{1}$ organizational climate measurement scale adapted by Chiang et $\mathrm{al}^{2,3}$ was used, and an instrument of Welfare and Work (UWES), a translation of the Schaufeli Instrument And Bakker ${ }^{4}$, Benevides-Pereira, A., Fraiz, D., PortoMartins, P., Spanish version. For the reliability analysis of both questionnaires, Cronbach's alpha was used.

The results obtained from the data analysis using the Spearman coefficient indicated that there is a significant statistical association between some dimensions of the organizational climate with some dimensions of worker engagement at both foundations, thus confirming the research hypothesis: "The greater the engagement among the workers of the foundations investigated presents an organizational climate more adequate for the achievement of the objectives of each institution". However, comparing the correlations of both samples, it can be observed that the correlations between the two institutions are not the same, so that no general conclusions can be drawn.

Key words: ORGANIZATIONAL CLIMATE AND ENGAGEMENT.

\section{INTRODUCCIÓN}

Correspondencia / Correspondence:

Ivonne Fuentealba Elgueta

Cura Gonzalo López № 1298, Los Lirios, Concepción

e-mail: ivonne.fuentealba@gmail.com

Tel.: +56974981328 / 41-2954452

Recibido: 02 de Abril de 2017 / Aceptado 23 de Julio de 2017

Hoy, más allá de ser un elemento de avance, el estudio del clima organizacional es algo necesario y fundamental para cualquier empresa, ya que es el indicador más preciso y demuestra los niveles que se tienen en la organización en cuanto a las relaciones laborales. $^{5}$

En efecto, aquellos empleados que tienden a experimentar estados emocionales positivos durante su jornada laboral, probablemente 
mostrarán mayor interés por su trabajo, lo cual generará mayores niveles de motivación y engagement. Por otra parte, mayores niveles de engagement llevarán al desarrollo de emociones positivas, generándose un espiral positivo ascendente. ${ }^{6}$

Lo anterior es necesario y fundamental para cualquier institución, pero por sobre todo para una de carácter social, como lo son las fundaciones donde se desarrolló el estudio.

La presente investigación surge por la necesidad de contar con antecedentes empíricos que aporten a la toma de decisiones institucionales para prever personal fidelizado, altamente motivado por su trabajo y en condiciones adecuadas para su ejercicio profesional, atendiendo a que éstos se constituyen en el eslabón más importante para dar cumplimiento a la misión de cada institución, las cuales se sustentan en los valores de solidaridad, justicia y equidad, entre otros. El presente estudio tiene por objetivo analizar la relación que existe entre el clima organizacional y el engagement en dos fundaciones, sin fines de lucro, de la Región del Bio Bio.

\section{MATERIAL Y MÉTODOS}

\section{Marco teórico}

\section{Clima Organizacional}

Analizando la información existente sobre clima organizacional se puede establecer que es un tema que ha venido tomando una relevancia importante durante el último tiempo; no obstante, sus orígenes son difusos, confundiéndose con otros conceptos tales como motivación, satisfacción y calidad de vida. De ahí la proliferación de teorias de estudios de medición e intervención, fusionados éstos a través de grandes perspectivas agrupadas en escuelas: la estructural, la perceptual, la interactiva y la cultural. ${ }^{7,8}$ La siguiente Tabla muestra algunas definiciones del constructo Clima Organizacional.

Tabla 1.

Definiciones de Clima Organizacional.

\begin{tabular}{|c|c|c|}
\hline Año & Autor & Aportes \\
\hline 1970 & $\begin{array}{l}\text { Campbell, } \\
\text { Dunnette, } \\
\text { Lawler y } \\
\text { Weick }\end{array}$ & $\begin{array}{l}\text { Conjunto de atributos especificos de una organización particular } \\
\text { que puede ser inducido por el modo como la organización se } \\
\text { enfrenta con sus miembros y con su entorno. Para el miembro } \\
\text { en particular de la organización, el clima toma la forma de un } \\
\text { conjunto de actitudes y de expectativas que describen las } \\
\text { caracteristicas estáticas de la organización, y las contingencias } \\
\text { del comportamiento-resultado y del resultado-resultado. El } \\
\text { clima son las percepciones individuales de las variables objetivas } \\
\text { y de los procesos organizacionales, pero es una variable } \\
\text { organizacional. }\end{array}$ \\
\hline 1975 & Schneider & $\begin{array}{l}\text { Percepciones o interpretaciones de significado que ayudan a los } \\
\text { individuos a tener conocimiento del mundo y saber cómo } \\
\text { comportarse. Las percepciones del clima son descripciones } \\
\text { psicológicamente fundamentales en las que hay acuerdo para } \\
\text { caracterizar las prácticas y procedimientos de un sistema. }\end{array}$ \\
\hline 1986 & $\begin{array}{l}\text { De Witte y } \\
\text { De Cock }\end{array}$ & $\begin{array}{l}\text { El clima representa una sintesis de percepciones sobre un } \\
\text { conjunto relativamente estable de orientaciones de valores de la } \\
\text { organización como un todo, que influye en el comportamiento } \\
\text { de los miembros de la organización respecto de la efectividad } \\
\text { organizacional que se centra en las políticas, prácticas y } \\
\text { procedimientos organizacionales formales e informales. }\end{array}$ \\
\hline 1988 & Rousseau & $\begin{array}{l}\text { Son las descripciones individuales del marco social o contextual } \\
\text { del cual forma parte la persona. }\end{array}$ \\
\hline 1990 & $\begin{array}{l}\text { Reichers y } \\
\text { Schneider }\end{array}$ & $\begin{array}{l}\text { Percepciones compartidas de politicas, prácticas y procedi- } \\
\text { mientos organizacionales, tanto formales como informales. }\end{array}$ \\
\hline
\end{tabular}

Koys y Decottis ${ }^{1}$ señalan que estudiar los climas en las organizaciones ha sido difícil debido a que se trata de un fenómeno complejo y con múltiples niveles; por ejemplo, actualmente la bibliografía existente debate sobre dos tipos de clima: el psicológico y el organizacional. El primero se estudia a nivel individual, mientras que el segundo se estudia a nivel organizacional. Ambos aspectos del clima son considerados fenómenos multidimensionales que describen la naturaleza de las percepciones que los empleados tienen de sus propias experiencias dentro de una organización.

Así también, puede que existan múltiples climas dentro de la misma organización, ya que la vida en la organización puede variar en cuanto a las percepciones de los miembros según los niveles de la misma, sus diferentes lugares de trabajo, o las diversas unidades dentro del mismo centro de trabajo ${ }^{9-13}$, citado en Chiang. ${ }^{2}$ Chiang et $\mathrm{al}^{2}$ efectúan una cronología de las definiciones que los investigadores han ofrecido para el clima, definiciones que denotan la elaboración del concepto desde las propiedades y características percibidas de la organización, discutidas por Forehand y Gilmer $^{14}$ y Friedlander y Margulies ${ }^{15}$; las representaciones e interpretaciones cognoscitivas de James y Jones ${ }^{16}$, de James y Sells ${ }^{17}$ y de Schneider ${ }^{18}$, a las percepciones generales o sumarias de Schneider y Reichers. ${ }^{19}$ El concepto recoge, entonces, desde las características de la organización determinadas como percepciones (donde se presume dominan los factores de organización 0 circunstanciales); los esquemas cognoscitivos (donde los factores individuales son primarios determinantes). Sin embargo, aparentemente, no existe investigación que trate si alguna de estas conceptualizaciones tiene un apoyo empírico mayor.

Chiang et $\mathrm{al}^{2}$ sostienen que el tratamiento del clima como percepción genérica de situaciones ha tenido la ventaja de permitir evaluaciones sumarias del contexto en investigaciones que, de otra manera, estarían focalizadas en gran parte en el nivel individual. Sin embargo, el clima como concepto tiene límites específicos que lo distinguen de otras características y de otras percepciones. Dos cualidades definidas y constantes del clima persisten en sus diversas conceptualizaciones: es una percepción y es descriptiva. Las percepciones son sensaciones o realizaciones experimentadas por un individuo. Las descripciones son informes de una persona de esas sensaciones.

En base a la acumulación de experiencia en una organización, las personas generan unas percepciones generales sobre ella. ${ }^{18}$ Estas percepciones sirven como mapa cognitivo del individuo sobre cómo funciona la organización y, por tanto, ayudan a determinar cuál es el comportamiento adecuado ante una situación dada. De esta manera, el clima es útil para adaptar el comportamiento del individuo a las exigencias de la vida en las organizaciones. ${ }^{19}$ Así, clima organizacional será entendido por el equipo investigador como las descripciones individuales del marco social o contextual del cual forma parte la persona, son percepciones compartidas de políticas, prácticas y procedimientos organizacionales, tanto formales como informales ${ }^{20,21}$, citado en Chiang et al. ${ }^{2}$

\section{Engagement}

El engagement se ha convertido en un concepto muy utilizado. Primero en el ámbito de los negocios y la consultoría y, recientemente, también en el ámbito académico. Las connotaciones diarias del engagement se refieren a la vinculación, la implicación, el compromiso, la pasión, el entusiasmo, el esfuerzo y la energía. Sin embargo, no existe acuerdo entre los profesionales sobre su significado y, por tanto, tampoco sobre su medida y evaluación. ${ }^{22}$ 
La psicología humanista sentó las bases para entender el engagement sobre la idea de la persona como centro de la organización que logrará una mayor identidad externa al reforzar el autoconcepto, lo que incidirá en el incremento de la comunicación, práctica colaborativa y resultados positivos en su área de trabajo, partiendo del autoconocimiento, determinación de sus valores y extensión de sus redes de influencia. ${ }^{23-25}$

Se han identificado relaciones del engagement con una mejor salud mental y física, mayor motivación intrínseca, autoeficacia, optimismo y autoestima de la organización. ${ }^{26-29}$

Además, los sujetos más comprometidos laboralmente muestran ser más capaces de apoyar a sus colegas, recibir retroalimentación y crear oportunidades de trabajo, adaptándose exitosamente a sus ambientes de trabajo ya que pueden reconocer, activar y generar recursos laborales con mayor facilidad. ${ }^{27,29}$

Salanova y Schaufeli ${ }^{22}$, distinguen "engagement del empleado" y "engagement en el trabajo (Work engagement)", aun cuando afirman que se utilizan normalmente como sinónimos. El engagement en el trabajo se refiere a la relación que tiene el trabajador con su trabajo, mientras que el engagement del empleado puede también incluir la relación existente con la organización. La presente investigación, cuando se refiere a engagement, lo hace haciendo alusión a engagement en el trabajo. La siguiente tabla muestra algunas definiciones del constructo Engagement.

Tabla 2.

Definiciones de Engagement.

\section{Año Autor Aportes}

1990 Kahn (citado Fue uno de los primeros autores en teorizar sobre el engagement, en Rodriguez-concibiéndolo como la energía concentrada que se dirige hacia Muñoz et al, metas organizacionales. 2013)

1997 Maslach y El engagement se caracteriza por energia, implicación y eficacia, Leiter (citado que son los opuestos directos de las tres dimensiones del en Lisbona et Burnout (medido con el MBI-GS): agotamiento, cinismo y falta al, 2009) de eficacia profesional, respectivamente.

2001 Shaufeli, El engagement es un estado mental positivo, satisfactorio y Salanova, relacionado al trabajo, caracterizado por vigor, dedicación y González- absorción. Más que un estado especifico y momentáneo, el Roma y engagement se refiere a un estado afectivo-cognitivo más Bakker, (citado persistente e influyente, que no está enfocado sobre un objeto, en Shaufelli y evento, individuo o conducta en particular. El vigor se caracteriza Bakker, 2003) por una gran voluntad de dedicar el esfuerzo al trabajo y la persistencia ante las dificultades. La dedicación se refiere a estar fuertemente involucrado en el trabajo y experimentar una sensación de entusiasmo, inspiración, orgullo, reto y significado. La absorción se caracteriza por estar totalmente concentrado y felizmente inmerso en el trabajo, de tal manera que el tiempo pasa rápidamente y se experimenta desagrado por tener que dejar el trabajo.

2007 Schaufeli y Es un estado psicológico de realización o la antítesis Salanova positiva del Burnout.

(citado en

Salanova y

Shaufelli,

2009)

2011 Salanova et Se observó que las emociones positivas poseen un efecto sobre al. (citado en los recursos positivos de las personas, tales como la creencia de Castellano et autoeficacia y engagement. Aparentemente el efecto positivo al, 2013) facilita conductas que promueven el engagement. Dichos autores observaron a partir de un estudio longitudinal de tres tiempos que las emociones positivas se asocian con el engagement. Concretamente se puede decir que las emociones positivas de alta activación (entusiasmo) poseen un mayor efecto predictivo sobre el engagement que las emociones positivas de baja activación (satisfacción y confort).

Fuente: Elaboración propia a partir de la revisión sistemática de la literatura.
El equipo investigador entenderá por Engagement: “estado mental positivo, satisfactorio y relacionado al trabajo, caracterizado por vigor, dedicación y absorción”. ${ }^{4}$

\section{Relación Clima Organizacional y Engagement}

En la revisión de la literatura efectuada no se encontraron investigaciones que relacionen el concepto de clima organizacional con Engagement. No obstante, se generaron hallazgos de la relación existente entre Burnout y Clima organizacional. Schaufeli y Salanova ${ }^{30,22,}$ plantean que el engagement en el trabajo es un estado psicológico de realización o la antítesis positiva del burnout. Se asume que contrariamente a aquellos que padecen burnout, los trabajadores engaged tienen un sentimiento de conexión energética y efectiva con su trabajo, en lugar de ver su trabajo como estresante y demandante lo perciben como retador. Para ellos, el trabajo es divertido y no una carga. En el engagement existe una sensación de realización en contraste al vacío de la vida que deja en la gente el sentimiento de pérdida característico del síndrome de burnout. ${ }^{22}$

Los trabajadores engaged tienen valores que coinciden con los que tiene la organización, mientras que en los trabajadores quemados se observan valores más contrapuestos con los valores de la organización. ${ }^{22}$

Se asume que el vigor y la dedicación son los polos opuestos del agotamiento y del cinismo, respectivamente. Estos dos últimos se consideran como los sintomas centrales del Burnout (Schaufeli y Taris). ${ }^{31,22}$ Comúnmente, los trabajadores quemados se sienten muy cansados y muestran una actitud distante hacia su trabajo, han agotado su energía y se identifican menos con su trabajo de lo que lo solían hacer. Por tanto, el burnout se caracteriza por una combinación de poca energía (agotamiento) y poca identificación (cinismo), mientras que el engagement en el trabajo se caracteriza por el patrón opuesto: una combinación de alta energía (vigor) y una elevada identificación (dedicación). Este marco teórico se ha confirmado de forma empírica en un estudio que demostró que el vigor y el agotamiento, así como la dedicación y el cinismo, constituyen dos dimensiones diferentes. ${ }^{32,22}$ Engagement incluye un componente conductual-energético (vigor), un componente emocional (dedicación) y un componente cognitivo (absorción). ${ }^{22}$ No se incluye en el concepto de engagement un constructo directamente opuesto al tercer componente del burnout -baja realización personal-, para lo cual existen dos razones: la primera es que la creciente evidencia empírica coloca al agotamiento emocional y el cinismo como elementos constitutivos del núcleo del burnout, mientras que la falta de eficiencia profesional parece tener un rol menos prominente. ${ }^{33}$ En segundo lugar, como resultado de entrevistas y discusiones con empleados y supervisores se encontró que el engagement está más particularmente caracterizado por el hecho de estar inmerso y felizmente dedicado al trabajo -un estado mental al cual llamamos absorción-. En este sentido la absorción es un aspecto distinto del engagement, que no se considera necesariamente opuesto a la ineficacia profesional. ${ }^{4}$

Por lo anterior, la relación entre Engagement y Clima Organizacional se sustenta en la relación existente entre Burnout y Clima Organizacional. Bettinardi, Montagner, Maini y Vidotto; Boada, De Diego y Argulló; Ogresta, Rusac y Zorec; Ramirez y Zurita; así como por Shelledy, Mikles, Mayo y Youtsey ${ }^{34-38}$, quienes manifiestan que en la percepción del Clima Organizacional, entre otras variables psicológicas, influyen en la aparición del Síndrome de Burnout. Ello también fue encontrado por Dávila y Romero ${ }^{39}$, 
quienes señalan en su estudio que, a mayor Síndrome de Burnout, será peor la percepción del clima organizacional. ${ }^{40}$

\section{METODOLOGÍA}

Se plantea como hipótesis de investigación, la siguiente:

"A mayor engagement entre los trabajadores de las fundaciones investigadas se presenta un clima organizacional más adecuado para el logro de los objetivos de cada institución"

El diseño metodológico del presente estudio corresponde a una investigación de campo, no experimental, transversal y descriptivo-correlacional.

El universo de estudio corresponde a 322 trabajadores de las fundaciones investigadas. Los roles que asumen las personas investigadas pueden ser directivo, profesional, técnico, administrativo o de servicio.

La muestra está compuesto por 237 trabajadores que respondieron el cuestionario, lo que equivale a un $73,6 \%$ del total del universo de estudio.

No se hizo consideración de ningún criterio de exclusión de los sujetos de la muestra. Los encuestados respondieron de forma voluntaria, por lo que el muestreo fue no probabilístico por conveniencia. Para la recolección de información se utilizaron los siguientes instrumentos:

1.- Clima organizacional: Escala de medición para clima organizacional de Koys y Decottis ${ }^{1}$, adaptación y validación de Chiang y otros. $^{2,3}$ Este instrumento consiste en 42 elementos. Los trabajadores y trabajadoras responden a cada elemento utilizando un formato de respuesta de Likert de cinco puntos (a saber: Muy de acuerdo $=5 ;$ De acuerdo $=4$; No estoy seguro $=3$; En desacuerdo $=2$; Totalmente en desacuerdo $=1$ ). Seis elementos expresados en palabras de forma negativa son valorados de forma inversa. Las

Tabla 3.

Información descriptiva para las ocho escalas de Clima Organizacional Koys y Decottis (1991).

\begin{tabular}{|c|c|c|}
\hline Escala & Descripción Escala & $\begin{array}{l}\mathrm{N}^{\circ} \text { de } \\
\text { items }\end{array}$ \\
\hline Autonomía & $\begin{array}{l}\text { Percepción del trabajador acerca de la autodeterminación } \\
\text { y responsabilidad necesaria en la toma de decisiones con } \\
\text { respecto a procedimientos del trabajo, metas y prioridades. }\end{array}$ & 5 \\
\hline Cohesión & $\begin{array}{l}\text { Percepción de las relaciones entre los trabajadores dentro } \\
\text { de la organización, la existencia de una atmósfera amigable } \\
\text { y de confianza y proporción de ayuda material en la realización } \\
\text { de las tareas. }\end{array}$ & 5 \\
\hline Confianza & $\begin{array}{l}\text { La percepción de la libertad para comunicarse abiertamente con } \\
\text { los superiores, para tratar temas sensibles o personales con la } \\
\text { confiancia suficiente de que esa comunicación no será violada o } \\
\text { usada en contra de los miembros. }\end{array}$ & 5 \\
\hline Presión & $\begin{array}{l}\text { La percepción que existe con respecto a los estándares de } \\
\text { desempeño, funcionamiento y finalización de la tarea. }\end{array}$ & 7 \\
\hline Apoyo & $\begin{array}{l}\text { La percepción que tienen los miembros acerca del respaldo y } \\
\text { tolerancia en el comportamiento dentro de la institución, esto } \\
\text { incluye el aprendizaje de los errores, por parte del trabajador, sin } \\
\text { miedo a la represalia de sus superiores o compañeros de trabajo. }\end{array}$ & 5 \\
\hline $\begin{array}{l}\text { Reconoci- } \\
\text { miento }\end{array}$ & $\begin{array}{l}\text { La percepción que tienen los miembros de la organización, con } \\
\text { respecto a la recompensa que reciben, por su contribución a } \\
\text { la empresa. }\end{array}$ & 5 \\
\hline Equidad & $\begin{array}{l}\text { La percepción que los empleados tienen acerca de si existen poli- } \\
\text { ticas y reglamentos equitativos y claros dentro de la institución. }\end{array}$ & 5 \\
\hline Innovación & $\begin{array}{l}\text { La percepción que se tiene acerca del ánimo que se tiene para } \\
\text { asumir riesgos, ser creativo y asumir nuevas áreas de trabajo, } \\
\text { en donde tenga poco o nada de experiencia }\end{array}$ & 5 \\
\hline
\end{tabular}

valoraciones de las escalas se obtienen mediante la suma total de los valores de los elementos de la escala. A continuación, se presenta información descriptiva para las ocho escalas de Clima Organizacional Koys y Decottis. ${ }^{1}$

2.-Engagement: Instrumento Utrecht Work Engagment Scale, UWES, el que está constituido por 17 items que abordaran sus tres dimensiones.

A continuación, se presenta información descriptiva para las tres escalas de Engagement.

Tabla 4.

Información descriptiva para las tres escalas de Engagement.

\begin{tabular}{llc} 
Escala & $\begin{array}{l}\text { Descripción Escala } \\
\text { Vigor }\end{array}$ & $\begin{array}{l}\text { Se caracteriza por una gran voluntad de dedicar el esfuerzo } \\
\text { al trabajo y la persistencia ante las dificultades. }\end{array}$ \\
$\begin{array}{ll}\mathrm{N}^{\circ} \text { dems } \\
\text { Dedicación }\end{array}$ & $\begin{array}{l}\text { Se refiere a estar fuertemente involucrado en el trabajo } \\
\text { y experimentar una sensación de entusiasmo, inspiración, } \\
\text { orgullo, reto y significado. }\end{array}$ & 5 \\
\hline Absorción & $\begin{array}{l}\text { Se caracteriza por estar totalmente concentrado y felizmente } \\
\text { inmerso en el trabajo, de tal manera que el tiempo pasa } \\
\text { rápidamente y se experimenta desagrado por tener que } \\
\text { dejar el trabajo. }\end{array}$ & 6 \\
\hline
\end{tabular}

Fuente: Elaboración propia.

Para el procesamiento de los datos que arrojará la totalidad de cuestionarios aplicados se utiliza el software SPSS versión 21, a través del cual se realizan los cálculos estadísticos, descriptivos y de relación.

\section{RESULTADOS}

\section{Resultados del desarrollo del instrumento de medición}

\section{Fiabilidad}

Analizar la fiabilidad de las dimensiones que componen un cuestionario es requerido para indicar en qué grado las respuestas a las preguntas (pertenecientes a una misma dimensión) covarian y se relacionan entre sí, lo que permite confirmar si los ítems miden lo mismo y son sumables en un puntaje total para cada variable. Las estimaciones de la consistencia interna de las escalas se calcularon utilizando el Coeficiente Alfa de Cronbach.

En la Tabla $N^{\circ} 5$ se observan los valores del alfa de Cronbach para la fiabilidad de los datos recogidos a través de la Encuesta de Clima Organizacional.

Para la dimensión "Equidad" que incluye 5 Ítems el coeficiente es

Tabla 5.

Fiabilidad Encuesta de Clima Organizacional.

$\begin{array}{llccccc}\begin{array}{l}\text { Factor de } \\ \text { clima } \\ \text { organizacional }\end{array} & \begin{array}{c}\mathrm{N}^{\circ} \\ \text { de } \\ \text { Items }\end{array} & \begin{array}{c}\text { Koys y } \\ \text { Decottis } \\ (1991)\end{array} & \begin{array}{c}\text { Chiang } \\ \text { y otros } \\ (2008)\end{array} & \begin{array}{c}\text { Chiag } \\ \text { y otros } \\ (2011)\end{array} & \begin{array}{c}\text { Actuales } \\ \text { resultados }\end{array} \\ \text { I } & \text { Apoyo } & 5 & 0,89-0,90 & 0,821 & 0,820 & 0,820 \\ \text { II } & \text { Autonomia } & 5 & 0,83-0,76 & 0,803 & 0,821 & 0,798 \\ \text { III } & \text { Cohesión } & 5 & 0,87-0,82 & 0,841 & 0,847 & 0,857 \\ \text { IV } & \text { Confianza } & 5 & 0,88-0,87 & 0,802 & 0,741 & 0,783 \\ \text { V } & \text { Equidad } & 5 & 0,82-0,82 & 0,639 & 0,725 & 0,603 \\ \text { VI } & \text { Innovación } & 5 & 0,80-0,87 & 0,861 & 0,835 & 0,860 \\ \text { VII } & \text { Presión } & 7 & 0,81-0,57 & 0,338 & 0,795 & -0,363 \\ \text { VIII } & \begin{array}{l}\text { Reconocimiento } \\ \end{array} & 5 & 0,83-0,84 & 0,700 & 0,814 & 0,698 \\ & \begin{array}{l}\text { Cuestionario } \\ \text { completo }\end{array} & 42 & & & & \\ & & & & & & \end{array}$

Fuente: Elaboración propia. 
0,502. No obstante, se decide eliminar la pregunta 23 pues aumenta considerar en el análisis de resultados $(0,603)$. En conclusión, la escala de este cuestionario arroja un coeficiente de rango de 0,603 a 0,860 , lo cual implica que es aceptable, por lo que es recomendable utilizar los Ítems para medir y analizar esta variable.

En la Tabla $N^{\circ} 6$ se observan los valores del alfa de Cronbach para la fiabilidad de los datos recogidos a través de la Encuesta de Engagement.

La escala de este cuestionario arroja un coeficiente de rango de 0,731

Tabla 6.

Fiabilidad Encuesta de Engagement.

\begin{tabular}{|c|c|c|c|c|c|c|}
\hline & $\begin{array}{l}\text { Factor de } \\
\text { Engagement }\end{array}$ & $\begin{array}{l}N^{\circ} \text { de } \\
\text { Items }\end{array}$ & $\begin{array}{c}\text { UWES } \\
-9\end{array}$ & $\begin{array}{c}\text { UWES } \\
-15\end{array}$ & $\begin{array}{c}\text { UWES } \\
-17\end{array}$ & $\begin{array}{c}\text { Actuales } \\
\text { resultados }\end{array}$ \\
\hline I & Absorción & 6 & 0,84 & 0,86 & 0,83 & 0,737 \\
\hline$\|$ & Dedicación & 5 & 0,89 & 0,92 & 0,92 & 0,820 \\
\hline III & $\begin{array}{l}\text { Vigor } \\
\text { Cuestionario completo }\end{array}$ & $\begin{array}{c}6 \\
17\end{array}$ & 0,79 & 0,82 & 0,80 & 0,731 \\
\hline
\end{tabular}

Fuente: Elaboración propia.

a 0,820, lo cual implica que para las escalas es aceptable, por lo que es recomendable utilizarlas para medir y analizar esta variable.

\section{Resultados de promedios}

Posterior a la descripción de la muestra, para referirse a los resultados obtenidos se dividió la muestra en las dos Fundaciones investigadas, entregando los resultados de la medición de Clima Organizacional y Engagement como se observa en las tablas $\mathrm{N}^{\circ} 7$ y 8 .

En las tablas $\mathrm{N}^{\circ} 7$ y $\mathrm{N}^{\circ} 8$ se observa que las fundaciones estudiadas poseen un alto nivel de engagement y una percepción favorable al logro de objetivos de las dimensiones que componen el clima organizacional.

\section{Resultados de correlaciones}

Para la correlación de las dimensiones analizadas en la Fundación 1, al aplicar el Coeficiente de Spearman se observó lo siguiente (Tabla $\mathrm{N}^{\circ}$ 9).

1. Correlación estadísticamente significativa entre la dimensión de Engagement Vigor y las dimensiones de Clima Organizacional Autonomía, Cohesión, Confianza, Apoyo, e Innovación.

2. Correlación estadísticamente significativa entre la dimensión de Engagement Dedicación y las dimensiones de Clima Organizacional Cohesión, Confianza, Apoyo, Reconocimiento, Equidad, e Innovación.

3. Correlación estadísticamente significativa entre la dimensión de Engagement Absorción y las dimensiones de Clima Organizacional Autonomía, Confianza, Reconocimiento, Equidad, e Innovación.

Para la correlación de las dimensiones analizadas en la Fundación 2, al aplicar el Coeficiente de Spearman se observó lo siguiente (Tabla $\left.\mathrm{N}^{\circ} 10\right)$ :

Tabla 7.

Promedio Dimensiones Encuesta Clima Organizacional Fundación 1 y Fundación 2.

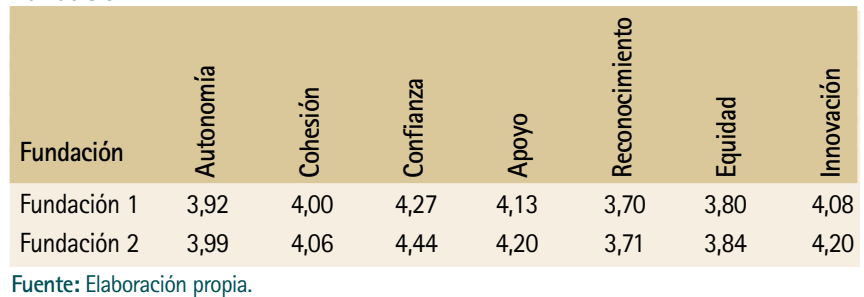

Fuente: Elaboración propia. la fiabilidad de la dimensión. La dimensión Presión no será posible

Tabla 8.

Promedio Dimensiones Encuesta Engagement Fundación 1 y Fundación 2.

$\begin{array}{lccc}\text { Fundación } & \text { Vigor } & \text { Dedicación } & \text { Absorción } \\ \text { Fundación } 1 & 4,77 & 4,97 & 4,40 \\ \text { Fundación } 2 & 5,04 & 5,24 & 4,49\end{array}$

Fuente: Elaboración propia.

Tabla 9.

Correlaciones (Rho de Spearman) de las dimensiones de Clima Organizacional y Engagement de la Fundación 1.

\begin{tabular}{|c|c|c|c|c|}
\hline & \multirow[b]{2}{*}{ Correlación } & \multicolumn{3}{|c|}{ Dimensiones de Engagement } \\
\hline & & Vigor & Dedicación & Absorción \\
\hline \multirow[b]{2}{*}{ Autonomía } & $\begin{array}{l}\text { Coeficiente } \\
\text { de Correlación }\end{array}$ & $0,644^{*}$ & 0,578 & $0,649^{*}$ \\
\hline & Sig. (2-tailed) & 0,044 & 0,08 & 0,042 \\
\hline \multirow[b]{2}{*}{ Cohesión } & $\begin{array}{l}\text { Coeficiente } \\
\text { de Correlación }\end{array}$ & $0,661^{*}$ & $0,644^{*}$ & 0,407 \\
\hline & Sig. (2-tailed) & 0,038 & 0,044 & 0,243 \\
\hline \multirow[b]{2}{*}{ Confianza } & $\begin{array}{l}\text { Coeficiente } \\
\text { de Correlación }\end{array}$ & $0,927^{* *}$ & $0,791^{* *}$ & $0,681^{*}$ \\
\hline & Sig. (2-tailed) & 0 & 0,006 & 0,03 \\
\hline \multirow[b]{2}{*}{ Presión } & $\begin{array}{l}\text { Coeficiente } \\
\text { de Correlación }\end{array}$ & 0,494 & 0,315 & $0,645^{*}$ \\
\hline & Sig. (2-tailed) & 0,147 & 0,376 & 0,044 \\
\hline \multirow[b]{2}{*}{ Apoyo } & $\begin{array}{l}\text { Coeficiente } \\
\text { de Correlación }\end{array}$ & $0,717^{*}$ & $0,868^{* *}$ & 0,561 \\
\hline & Sig. (2-tailed) & 0,02 & 0,001 & 0,092 \\
\hline \multirow[b]{2}{*}{ Reconocimiento } & $\begin{array}{l}\text { Coeficiente } \\
\text { de Correlación }\end{array}$ & 0,588 & $0,914^{* *}$ & $0,833^{* *}$ \\
\hline & Sig. (2-tailed) & 0,074 & 0 & 0,003 \\
\hline \multirow[b]{2}{*}{ Equidad } & $\begin{array}{l}\text { Coeficiente } \\
\text { de Correlación }\end{array}$ & 0,608 & $0,775^{* *}$ & $0,735^{*}$ \\
\hline & Sig. (2-tailed) & 0,062 & 0,008 & 0,015 \\
\hline \multirow[b]{2}{*}{ Innovación } & $\begin{array}{l}\text { Coeficiente } \\
\text { de Correlación }\end{array}$ & $0,758^{*}$ & $0,890^{* *}$ & $0,699^{*}$ \\
\hline & Sig. (2-tailed) & 0,011 & 0,001 & 0,024 \\
\hline
\end{tabular}

Tabla 10.

Correlaciones (Rho de Spearman) de las dimensiones de Clima Organizacional y Engagement de la Fundación 2.

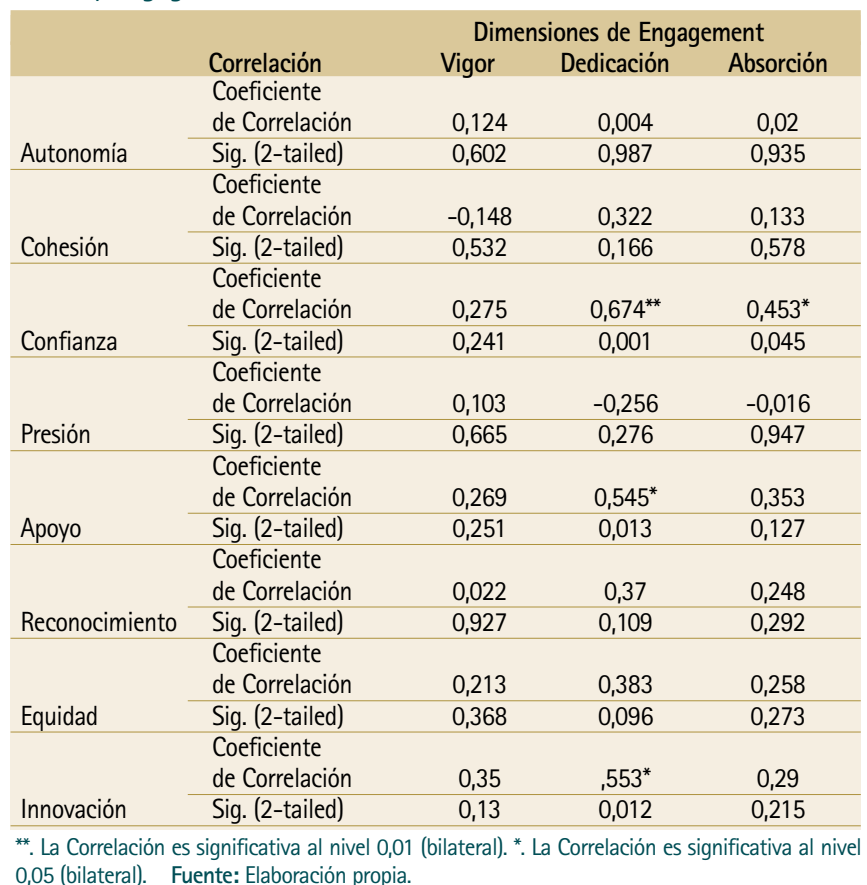


1. Correlación estadísticamente significativa entre la dimensión de Engagement Dedicación y las dimensiones de Clima Organizacional Confianza, Apoyo e Innovación.

2. Correlación estadísticamente significativa entre la dimensión de Engagement Absorción y la dimensión de Clima Organizacional Confianza.

\section{CONCLUSIONES}

El Clima Organizacional es la descripción individual del marco social o contextual del cual forma parte una persona; son percepciones compartidas de políticas, prácticas y procedimientos organizaciones tanto formales como informales. Se inscribe dentro de una teoría cognitiva general de la conducta, en la que las percepciones de los individuos tienen consecuencias reales sobre las situaciones y comportamientos.

El Engagement es el estado mental positivo, satisfactorio y relacionado al trabajo, caracterizado por vigor, dedicación y absorción. El Engagement se sustenta en el modelo de demandas y recursos laborales, el cual plantea que ante las demandas laborales puede surgir una situación de estrés en los trabajadores, de manera que de no lograr afrontar se produce agotamiento que lleva al bournout; en caso contrario, quienes logran responder a tales exigencias laborales a través de los recursos laborales se inician en un proceso motivacional que conduce al Engagement.

Del análisis de las variables Engagement y Clima Organizacional, y de la relación entre ellas, se desprenden las siguientes conclusiones:

\section{El Engagement de los trabajadores de cada Fundación}

- En la Fundación 1, se puede concluir que los trabajadores que la integran poseen un alto grado de Engagement, pues presentan promedios iguales o superiores a 4,40, donde las alternativas a las afirmaciones que dan a conocer la presencia de engagement son: 4 = bastantes veces; 5 = casi siempre; $\mathrm{y}, 6=$ siempre. De lo anterior se puede inferir que, en orden de prelación, se sienten involucrados en el trabajo y experimentan una sensación de entusiasmo, inspiración, orgullo, reto y significado, gran voluntad de dedicar esfuerzo al trabajo y persistencia ante las dificultades, concentrados y felizmente inmersos en su trabajo, de tal manera que el tiempo pasa rápidamente y experimentan desagrado por tener que dejarlo.

- En la Fundación 2, se puede concluir que los trabajadores que la integran poseen un alto grado de Engagement, pues presentan promedios iguales o superiores a 4,49. De lo anterior se puede inferir que, en orden de prelación, se sienten involucrados en el trabajo y experimentan una sensación de entusiasmo, inspiración, orgullo, reto y significado, gran voluntad de dedicar esfuerzo al trabajo y persistencia ante las dificultades, concentrados y felizmente inmersos en su trabajo, de tal manera que el tiempo pasa rápidamente y experimentan desagrado por tener que dejarlo.

- De lo antes expuesto se puede concluir que ambas instituciones presentan Engagement, con un grado de intensidad mayor para cada dimensión en la Fundación 2.

\section{Con respecto al Clima Organizacional de los trabaja- dores de cada Fundación}

- En la Fundación 1, se puede concluir que los trabajadores que la integran poseen una percepción del Clima Organizacional favorable al logro de objetivos en las dimensiones: Cohesión, correspondiente a la percepción de las relaciones entre los trabajadores dentro de la Fundación, caracterizada por una atmósfera favorable de confianza y de ayuda material en la realización de las tareas; Innovación: correspondiente a la percepción del ánimo que se tiene para asumir riesgos, ser creativos, asumir nuevas áreas de trabajo contando con poca o nada de experiencia; Apoyo, correspondiente a la percepción que tienen los trabajadores acerca del respaldo y tolerancia en el comportamiento de la Fundación; Confianza, correspondiente a la percepción de la libertad que tienen los trabajadores para comunicarse abiertamente con sus superiores.

- En la Fundación 2, se puede concluir que los trabajadores que la integran poseen una percepción del Clima Organizacional favorable al logro de objetivos en las dimensiones: Confianza, correspondiente a la percepción de la libertad que tienen los trabajadores para comunicarse abiertamente con sus superiores; Apoyo, correspondiente a la percepción que tienen los trabajadores acerca del respaldo y tolerancia en el comportamiento de la Fundación; Innovación: correspondiente a la percepción del ánimo que se tiene para asumir riesgos, ser creativos, asumir nuevas áreas de trabajo contando con poca o nada de experiencia; Cohesión, correspondiente a la percepción de las relaciones entre los trabajadores dentro de la Fundación, caracterizada por una atmósfera favorable de confianza y de ayuda material en la realización de las tareas.

- De lo antes expuesto se puede concluir que ambas instituciones presentan dimensiones de clima percibidas como favorables al logro de objetivos.

\section{Con respecto a la relación del Engagement y el Clima Organizacional de los trabajadores de ambas Fundaciones}

- Se observa que existe para ambas fundaciones entre las dimensiones confianza y dedicación.

- Se observó una mayor presencia de relaciones entre las dimensiones estudiadas, en la Fundación 1 que en la Fundación 2. Se infiere que entre las razones que pudieran describir este comportamiento se encuentran las acciones concretas en materia de favorecer un clima organizacional promotor del trabajo al interior de la Fundación 1, no así en la Fundación 2. Además de la diferencia de años de constitución de cada una.

- Para la Fundación 1 se observa una correlación positiva de la dimensión Vigor del Engagement con las dimensiones Confianza, Innovación, Apoyo, Cohesión y Autonomía del Clima Organizacional. De lo anterior se puede inferir que la voluntad de los trabajadores de dedicar esfuerzo a su trabajo y persistir ante dificultades está determinada en orden de prelación por su percepción de la libertad que poseen para comunicarse abiertamente con sus superiores, la que tienen acerca del ánimo para asumir riesgos, del respaldo y tolerancia de su comportamiento dentro de la institución, de sus relaciones con los otros trabajadores y la autodeterminación y responsabilidad para tomar decisiones.

- Para la Fundación 1 se observa una correlación positiva de la dimensión Dedicación del Engagement con las dimensiones Reconocimiento, Innovación, Apoyo, Confianza, Equidad y Cohesión del Clima Organizacional. De lo anterior se puede inferir que el nivel de implicación en el trabajo está determinada en orden de prelación por la percepción que tienen los trabajadores con respecto a las recompensas que reciben por su contribución a la fundación, por el ánimo para asumir riesgos, por la tolerancia a su comportamiento dentro de la fundación, por la 
libertad de comunicarse abiertamente con sus superiores, por las políticas reconocidas dentro de la institución y por las relaciones que mantienen con el resto de los trabajadores de la fundación.

- Para la Fundación 1 se observa una correlación positiva de la dimensión Absorción del Engagement con las dimensiones Reconocimiento, Equidad, Innovación, Confianza y Autonomía del Clima Organizacional; de lo anterior, se puede inferir que el sentirse feliz inmerso en el trabajo está determinado por la percepción que tienen los trabajadores respecto a la recompensas que reciben por su contribución a la fundación, por las políticas implementadas, por el ánimo que se tiene para asumir riesgos, por la libertad para comunicarse abiertamente con sus superiores y por su autodeterminación en la toma de decisión.

- Para la Fundación 2 se observa una correlación positiva de la dimensión Dedicación del Engagement con las dimensiones Confianza, Innovación y Apoyo del Clima Organizacional; de lo anterior, se puede inferir que el nivel de implicación en el trabajo está determinado en orden de la prelación por la percepción que tienen los trabajadores de comunicarse abiertamente con sus superiores, por el ánimo para asumir riesgos, por el respaldo y tolerancia frente a su comportamiento.

- Para la Fundación 2 se observa una correlación positiva de la dimensión Absorción del Engagement con la dimensión Confianza del Clima Organizacional; de lo anterior, se puede inferir que el sentirse feliz inmerso en el trabajo está determinada por la percepción que tienen los trabajadores respecto a la libertad de comunicarse abiertamente con sus superiores.

De lo anteriormente expuesto, se puede concluir que las fundaciones estudiadas poseen un alto nivel de engagement y una percepción favorable al logro de objetivos de las dimensiones que componen el clima organizacional, lo que se constituye en una característica ampliamente positiva para ellas, atendiendo a que son instituciones promotoras de las personas y su dignidad. Lo anterior hace de estas instituciones organizaciones coherentes entre su accionar y la alta carga valórica que las caracteriza, que pretenden instalar en sí mismas y a través de su acción valores como la justicia, la dignidad y la equidad. Para lo anterior, claramente es necesario contar con personas que no sólo fidelicen con estos valores, sino que los hagan propios y que el trabajo que desarrollan los realice plenamente, generando adecuados procesos interventivos que potencien a los individuos atendidos. Trabajadores con alto grado de engagement aseguran individuos que se apasionan por su trabajo y que se motivan a efectuar con alegría y entusiasmo el mismo, constituyéndose así en promotores de cambio en sí mismos. Trabajadores con una percepción adecuada al logro de objetivos de las dimensiones del clima organizacional demuestran relaciones laborales sanas.

Los resultados obtenidos en esta investigación permiten concluir que existe asociación estadística significativa entre algunas dimensiones del clima organizacional con algunas dimensiones de engagement de los trabajadores de la Fundación 1 y Fundación 2, comprobando así la hipótesis planteada de investigación: "A mayor engagement entre los trabajadores de las fundaciones investigadas se presenta un clima organizacional más adecuado para el logro de los objetivos de cada institución". No obstante lo anterior, analizando las correlaciones de ambas muestras, se observa que las correlaciones que se dan entre una y otra institución no son las mismas, por lo que no se pueden realizar conclusiones generales.

\section{REFERENCIAS}

1. Koys D, Decottis T. Inductive Measures of Psychological Climate. Hum Relat. 1991; 44(3):265-385.

2. Chiang $M$, Salazar $C$, Huerta $P$, Nuñez A. Clima organizacional y satisfacción laboral en organizaciones del sector estatal (instituciones públicas) desarrollo, adaptación y validación de instrumentos. Universum. 2008; 23(2):66-85.

3. Chiang M, Salazar M, Martín M, Nuñez A. Clima organizacional y satisfacción laboral: Una comparación entre hospitales públicos de alta y baja complejidad. Salud trab. 2011; 19(1):5-16.

4. Schaufeli W, Bakker A. UWES - Ultrecht Work Engagement Escale. Preliminary Manual. Ultrech: Occupational Health Psychology Unit Ultrech University; 2003.

5. Peña $M$, Díaz $M$, Olivares $M$. Diagnóstico del clima organizacional promotor de estrategias gerenciales en las pequeñas empresas de la industria metalmecánica. RIAF. 2015; 8(5):15-24.

6. Castellano E, Cifre E, Spontón C, Medrano L, Maffei L. Emociones positivas y negativas en la predicción del Burnout y engagement en el trabajo. Rev Per Psic y Trab Soc. 2013; (2):75-88.

7. Moran $E_{1}$ Volkwein J. The cultural approach to the formation of organizational climate. Hum Relat. 1992; 45(1):19-47.

8. Cardona $D$, Zambrano R. Revisión de instrumentos de evaluación de clima organizacional. Estudios Gerenciales. 2014; (30):184-189.
9. Johnston $\mathrm{H}$. A new conceptualization of source of organizational climate. Admin Sci Quart. 1976; 21(1):95-103.

10. Litwin G, Stringer R. Motivación y Clima Organizacional. Cambridge: Harvard University; 1968.

11. Payne R, Mansfield R. Correlates of individual perceptions of organizational climate. J Occup Psychol. 1978; 51(3):209-218.

12. Powell G, Butterfield D. The case for subsystem climates in organizations. Acad Manage Rev. 1978; 3(1):151-157.

13. Schneider B, Hall D. Toward spsifying the concept of work climate: A study of romant catholic diocesan priests. J Appl Psychol. 1972. 56(6):447-455.

14. Forehand G, Gilmer B. Environmental variation in studies of organizational behaviour. Psychol Bull. 1964 Dec; 62:361-382.

15. Friedlander F, Margulies N. Multiple Impacts of Organizational Climate and Individual Value Systems upon Job Satisfaction. Pers Psychol. 1969; (22):171-183.

16. James $L$, Jones A. Organizational Climate: A Review of Theory and Research. Psychol Bull. 1974; 81(12):1096-1112.

17. James $L$, Jones A. And Organizational Model: Componets and Measurement. Men in Social Systems: Results of a Three Year Multiorganizational Study. New Orleans: APA;1974.

18. Schneider B. Organizational climates: An Essay. Person Psychol. 1975; 28(4):447-479. 
19. Schneider B, Reichers A. On the etiology of climates. Person Psychol.1983; 36(1): 19-39.

20. Rousseau DM. The Construction of Climate in Organizational Research. In: Cooper C, Robertson I, editors. International Review of Industrial and Organizational Psychology. Chichester: Wiley; 1988. p.139-158.

21. Reichers A, Schneider B. Climate and Culture: An Evolution of Constructs. Organizational Climate and Culture. San Francisco: Jossey-Bass; 1990.

22. Salanova $M$, Schaufeli W. El engagement en el trabajo: cuando el trabajo se convierte en pasión. Madrid: Alianza; 2009.

23. Cárdenas $T$, Jaik A. Engagement Ilusión por el trabajo Un modelo teóricoconceptual. México: Red Durango de investigadores Educativos A.C.; 2014.

24. Ciófalo-Lagos M, Dionne-Duddy G, Ramírez-Vukovich R. El autoconcepto a partir del diálogo apreciativo centrado en la persona en conversaciones cara a cara. Bloomington: Palibrio; 2013.

25. Reynoso-Nuñez C, Ciófalo-Lagos M. Socialización y engagement. Global Conference on Business and Finance; 2015 Jan 4-7; Las Vegas, USA. Las Vegas: The Institute for Business and Finance Research; 2015.

26. Sappala $P$, Merino S, Feldt $T$, Hakanen J, Kinnunen U, Tolvanen A, Schaufeli $W$. The constructor validity of the Ultrecht Work Engagement Scale: Multisample and longitudinal evidence. J Happiness Stud. 2009; 10(4):459481.

27. Xanthopoulou D, Bakker A, Demerouti E, Schaufeli W. Reciprocal relationships between job resources, personal resources, and work engagement. J Vocat Behav. 2008; 74(3):235-244.

28. Xanthopoulou D, Bakker A, Demerouti E, Schaufeli W. Work engagement and financial returns: A diary study on the role of job and personal resources. J Occup Organ Psychol. 2009; 82(1):183-200.

29. Müller R, Pérez C, Ramirez L. Estructura factorial y consistencia interna de la UTRECH WORK ENGAGEMENT SCALE (UWES) 17 entre trabajadores sanitarios de Chile. Liber. 2013; 19(2):163-171.

30. Schaufeli W, Salanova M. Work Engagement: an emerging psychologycal concept and its implications for organizations. In: Gilliland SW, Steiner DD, Skarlicki DP, editors. Managing social and ethical Issues in organizations. Greenwich: IAP; 2007.

31. Schaufeli W, Bakker A, Taris T. The conceptualization and measurement of burnout: Common ground and words apart. Work Stress. 2005; 19(3):256262.

32. González-Roma V, Schaufeli W, Bakker A, Llorent S. Burnout and engagement: Independent factors or opposite poles?. J Vocat Behav. 2006; 68(1):165-174.

33. Maslach C, Shaufeli W, Leiter M. Job burnout. Annu Rev Psychol. 2001; 52: 397-422.

34. Bettinardi 0 , Montagner V, Mini M, Vidotto G. Clima organizacional, la confianza y el desgaste en un centro de rehabilitación. G Ital Ergon Lav Med. 2008; 30(1 Suppl A):59-63.

35. Boada J, De Diego R, Argulló E. El Burnout y las manifestaciones psicosomáticas como consecuencias del Clima Organizacional y de la motivación laboral. Psicothema. 2004;(16):132-138.
36. Ogresta J, Rusac S, Zorec L. Relación entre el Síndrome de Burnout y la satisfacción laboral entre los trabajadores de la salud mental. Croat Med J. 2008; 49(3):364-374.

37. Ramírez $M$, Zurita R. Variables organizacionales y psicosociales asociadas al Sindrome de Burnout en trabajadores del ámbito educacional. Polis. 2010; 9(23):512-534.

38. Schelledy $D$, Mikles $S$, Mayo $D$, Youtsey J. Análisis de la satisfacción laboral, el Burnout, y la intención de las vías respiratorias profesionales de la atención a abandonar el campo o el trabajo. Cuidado Respiratorio. 1992; 37:46-60.

39. Dávila J, Romero P. Relación entre el Sindrome de Burnout y la percepción del clima organizacional en médicos del área de urgencias [tesis]. Caracas: Universidad Católica Andrés Bello; 2010.

40. Moreno A, Hidalgo M. Relación ente el Clima Organizacional y el Sindrome de Burnout en empleados de una firma de vigilancia en una empresa carbonera de la Guajira, Colombia. Psicogente. 2010; 13(24):292-305.

\section{Referencias en tablas y complementarias:}

Campbell J, Dunnette M, Lawler E, Weick K. Managerial Behavior: Performance and Effectiveness. Nueva York: McGraw-Hill;1970.

Chiang $M$, Martín $M$, Nuñez A. Relaciones entre clima organizacional y la satisfacción laboral. Madrid: Universidad Pontificia Comillas; 2010.

De Witte K, De Cock G. Organizational Climate: Its Relations - hip with Managerial, Activities and Communication Structures, In: Debus G, Schroiff $\mathrm{H}$, editors. The Psychology of Work and Organizations; Current Trends and Issues. Amsterdam: Elsevier; 1986.

James L, Sells S. Psychological Climate: Theoretical perpectives and empirical research. In: Magmussom D, editor. Toward a psychology of situations: An interactional perspective. New Jersey: Lawrence Erlbaum Associates; 1981. p. 275-295.

Kahn W. Psychological conditions of personal engagement and disengagement at work. Acad Manage J. 1990; 33(4):692-724.

Lisbona A, Morales J, Palací F. El engagement como resultado de la socialización organizacional. Rev Int Psicol Ter Psicol. 2009; 9(1):89-100.

Rodríguez-Muñoz A, Bakker A. Engagement en el trabajo. En: Moreno-Jiménez $B$, Garrosa E, editores. Salud Laboral riesgos laborales psicosociales y bienestar laboral. Madrid: Edics. Pirámide; 2013.

Salanova M, González J, Baker A. UWES: Ultrech Work engagement Scale. Escala de Engagement en el Trabajo de Utrecht Occupational Health Psychology Unit. Utrecht: Utrecht University; 2002.

Salanova M, Schaufeli W, Llorens S. Yes, I Can, I Feel Good, and I Just Do It! On Gain Cycles and Spirals of Efficacy Beliefs, Affect, and Engagement. Appl Psychol. 2011; (60): 225-285.

Schaufeli W, Salanova M, González-Romá V, Bakker A.The measurement of engagement and burnout: A confirmative analytic approach. J Happiness Stud. 2002; 3(1):71-92.

Schaufeli W, Bakker A. Escala Ultrech de Engagement en el Trabajo (Valdéz H, Ron C, traductores UWES-Ultrecht work engagement scale; 2003). México: Comisaría General de Prevención y Reinserción Social del Estado de Jalisco; 2011. 